\title{
In situ loss measurement of direct UV written waveguides using integrated Bragg gratings
}

\author{
Helen L Rogers, "Sumiaty Ambran, Christopher Holmes, \\ Peter G R Smith and James C Gates \\ Optoelectronics Research Centre, University of Southampton, Highfield, Southampton, \\ Hampshire, SO17 1BJ, UK \\ *Corresponding author: hlrlg09@orc.soton.ac.uk
}

The propagation loss of a direct UV written silica-on-silicon waveguide is measured using an elegant non-destructive method. The technique uses integrated Bragg grating structures, which are observed from opposing launch directions to obtain information about the optical power at different positions along the length of the waveguide. Critically the technique is ratiometric and independent of coupling loss and grating variability. This high precision measurement is suitable for low-loss planar waveguides. From this data, the propagation loss of the UV written waveguides was observed to be $0.235 \pm 0.006 \mathrm{~dB} / \mathrm{cm}$.

OCIS codes: $230.1480,230.7370$.

Measuring the attenuation of an integrated or planar waveguide has many associated difficulties stemming from both the typically low propagation losses and the short lengths of the waveguides. An effective measurement technique must determine the attenuation loss in the presence of the additional losses occurring when light is coupled into and out of the waveguide. These additional losses are the fractional coupling coefficient due to modal mismatch, scattering 
loss at the imperfect interface and the Fresnel reflections. We present a novel measurement technique capable of sensitive loss discrimination that is independent of these additional factors, using integrated Bragg grating structures in a non-destructive manner. Importantly the method is ratiometric and does not require any physical changes that can affect power measurements such as variation in output facet as in cutback measurements.

For optical fibers the cutback approach is easy to use, simply involving cleaving the fiber into shorter lengths by meters or even kilometers as appropriate, to provide sufficient loss difference to be clearly distinguishable above variability due to cleaving. However the situation in integrated optics is more complex.

In integrated optics, the crudest cutback approach involves multiple cut and polish steps to determine the propagation loss of the waveguide [1]. Aside from the inconvenience, the method suffers from two principle drawbacks. The first is the dependence of the measurement on the coupling efficiency. The multiple polishing steps will not provide consistent end facets, changing the scattering loss and modal transmission with each subsequent cutback thereby increasing error in the resulting loss calculation. The second drawback, and source of error is a consequence of the small total loss associated with a short waveguide. For a low-loss waveguide, the cutback technique will not observe a total loss sufficient to distinguish between Fresnel reflection losses, coupling losses and the propagation loss.

There are many other methods which can be used to measure propagation losses within integrated optical waveguides, but each suffers from at least one of the errors mentioned above. For example, the prism coupling method [2] measures the light coupled out of a waveguide at two points along its length, and the values are compared to obtain propagation loss data, while the scattered light measurement method [3] involves imaging the light scattered out of the 
waveguide along its length. Both prism coupling and scattered light methods are suitable for waveguides with large losses (around $1 \mathrm{~dB} / \mathrm{cm}$ or higher); while lower loss waveguides do not lose enough light in the direction of measurement for accurate propagation loss data to be obtained. Furthermore, the prism coupling method is also sensitive to variability in coupling efficiency each time the prism is moved. The Fabry-Perot method [4] involves measuring the finesse of a Fabry-Perot cavity constructed around the waveguide. This method has a dependency on the coupling efficiency into and out of the waveguide, and works best for higher index materials such as $\mathrm{LiNbO}_{3}$. The optimum end-fire coupling method [5] overcomes the coupling efficiency issues, but will again have issues resolving the attenuation of low-loss waveguides, due to the small intensity changes involved.

The technique described in this letter involves using integrated Bragg grating structures to perform reflectivity measurements in a variation of the cutback approach. The loss is measured between a series of gratings by observing the reflected spectra from opposing launch directions. By conducting the measurement in this way there is no dependence on the coupling losses to the waveguide, and the propagation loss of waveguide with low losses can be resolved. As all other losses are accounted for, this method provides a direct method of measuring the attenuation of a waveguide. In addition the technique is also independent of the actual grating strength provided the reflected signal is sufficient to determine the reflected power.

The method was developed using silica-on-silicon samples produced by flame hydrolysis deposition (FHD). The core layer was made photosensitive by the addition of germanium and boron dopants within the flame [6]. The planar cladding and core layers produced are all index matched, and the samples were hydrogen loaded before UV writing to further promote 
photosensitivity. Similar samples produced by plasma enhanced chemical vapor deposition (PECVD) have a reported propagation loss of $<0.2 \mathrm{~dB} / \mathrm{cm}[5]$.

The direct grating writing technique [6] produces direct UV written waveguides with the option of simultaneously defined Bragg grating structures. A schematic of the waveguides produced for the loss measurement can be seen in figure 1. Each waveguide contains a number of Bragg gratings of different periods along the length of the waveguide. The waveguides were $40 \mathrm{~mm}$ long and contained either five $2 \mathrm{~mm}$ long gratings with $6 \mathrm{~mm}$ spacing, or ten $2 \mathrm{~mm}$ long gratings with $2 \mathrm{~mm}$ long spacing. Fluence-matching the gratings and waveguides ensures the presence of the gratings does not influence the waveguide loss. Light from an amplified spontaneous emission source was launched into each end of the sample in turn via a polarizer (optimized for the TE mode in our case) and circulator and the reflection spectrum obtained using an Optical Spectrum Analyzer (OSA). The reflected spectra obtained from the waveguide containing ten gratings can be seen in figure 2 .

Theoretically, the power reflected by the $i^{\text {th }}$ grating from each direction can be modeled as follows, as shown in figure 3 ;

$$
\begin{gathered}
R_{i}^{\prime}=t^{\prime 2} r_{i} \exp ^{\left(-2 \alpha x_{i}\right)} \\
R_{i}^{\prime \prime}=t^{\prime \prime 2} r_{i} \exp ^{\left(-2 \alpha\left(L-x_{i}\right)\right)}
\end{gathered}
$$

where $R_{i}{ }^{\prime}$ and $R_{i}{ }^{\prime}$ are the reflected powers from grating $i$ in opposing directions, $t^{\prime}$ and $t^{\prime \prime}$ are the launched power in opposing directions taking into account the coupling losses, $L$ is the length of the waveguide, $x_{i}$ is the position of the $i^{\text {th }}$ grating within the waveguide, $\alpha$ is the loss of the waveguide (in units of $\mathrm{cm}^{-1}$ ) and $r_{i}$ is the reflectivity of grating $i$. Taking the ratio of the reflected power we obtain;

$$
\frac{R_{i}^{\prime}}{R_{i}^{\prime \prime}}=\eta \exp ^{\left(-4 \alpha x_{i}\right)}, \quad \eta=\frac{t^{\prime 2}}{t^{\prime 2}} \exp ^{(2 \alpha L)}
$$


Taking the logarithm of this equation we obtain

$$
\ln \frac{R_{i}^{\prime}}{R_{i}^{\prime \prime}}=\ln \eta-4 \alpha x_{i}
$$

showing the loss $\alpha$ of our waveguide can be obtained directly from the gradient of a plot of the logarithm of reflected power $\left(\mathrm{R}_{\mathrm{i}}^{\prime} / \mathrm{R}_{\mathrm{i}}{ }^{\prime \prime}\right)$ against position $x_{i}$. This simple relation shows that the measurement technique provides a value for the propagation loss which is independent of the coupling losses associated with the characterization set up.

In practice, as powers are measured in $\mathrm{dB}$, and using $\gamma=4.34 \alpha$, where $\gamma$ is the loss in $\mathrm{dB} / \mathrm{cm}^{-1}$, we may simply plot a graph of the difference of the $\mathrm{dB}$ power of the reflectivity from each direction as a function of the position of the grating along the waveguide, yielding the plot shown in figure 4 . The gradient of this plot gives an attenuation $(\alpha)$ of $0.235 \pm 0.006 \mathrm{~dB} / \mathrm{cm}$ for the waveguide containing 10 gratings. The equivalent plot for a different waveguide containing 5 gratings is shown in figure 5. This shows a similar loss $(0.226 \pm 0.026 \mathrm{~dB} / \mathrm{cm})$ which demonstrates that the gratings contribute no significant additional loss to the waveguide. This is consistent with weak type I Bragg gratings which show no transmission loss outside the bandwidth of the grating response [7]. The errors are determined via regression and intrinsically give a robust value for the uncertainty of the loss, where other authors can only give values for repeatability. In these experimental results our measured standard error is around ten times better than previously reported techniques $[2,4]$.

To perform these measurements a number of assumptions are made about the waveguide and Bragg gratings. The first is that the waveguides are of sufficient quality to support Bragg grating structures. Secondly, the gratings are assumed to be weak, so that the reflection spectrum is identical when measured from opposing directions. Importantly, the physical position of each grating is known very accurately (within a few microns) due to the direct UV writing approach, 
and as each grating is identical, other than in its unique period, we can be confident that the reflection wavelengths correspond to gratings at very well known physical positions. A further assumption is that the grating spectra reflectivities from a particular direction are all measured at the same time so that coupling efficiency is constant. We also require the source spectrum to be the same for the measurements from each direction although it does not need to be spectrally uniform. Furthermore, we assume that the coupling is wavelength independent over the wavelength range in use, which is typical of facet launched waveguides. In practice all of these are easy to achieve; the grating strength is controlled during writing, the spectra of all gratings are conveniently taken at the same time using an OSA guaranteeing consistent coupling. In addition we have assumed that the losses are constant along the length of the waveguide, this is corroborated by the small uncertainty of the measurement.

This method provides loss measurements which are independent of coupling efficiency, facet reflectivity and Fresnel reflection loss. The technique can potentially be applied to many photosensitive waveguides, such as chalcogenide glasses, and flat fiber [8]. The use of integrated Bragg grating structures allows the measurement to be carried out on any device with two or more integrated gratings, so accurate propagation loss measurements of s-bends, cross-couplers and other waveguide geometries can be achieved. The technique also allows polarization dependent attenuation of single mode waveguides to be measured, making use of the polarizer within the setup. An additional application is to measure the losses of different modes within multi-mode waveguides, so long as higher order modes can be distinguished and identified. The measurements also allow regression analysis of the data providing a robust uncertainty of the loss measurement.

\section{Acknowledgement}


This research was funded by EPSRC, UK. 


\section{References}

1. A. Ghatak and K. Thyagarajan, "Measurement of attenuation," in Introduction to Fiber Optics, (Cambridge University Press, 1997), pp. 413-416.

2. Y.H. Won, P.C. Jaussaud and G.H. Chartier, "Three Prism loss measurements of optical waveguides," Appl. Phys. Lett. 37, 269-271 (1980).

3. Y. Okamura, S. Yoshinaka and S. Yamamoto, "Measuring mode propagation losses of integrated optical waveguides: a simple method," Appl. Optics. 22, 3892-3894 (1983).

4. T. Feuchter and C. Thirstrup, "High-Precision Planar Waveguide Propagation Loss Measurement Technique Using a Fabry-Perot Cavity,” IEEE Photon. Tech. Lett. 6, 12441247 (1994).

5. M. Haruna, Y. Segawa and H. Nishihara, "Nondestructive and simple method of opticalwaveguide loss measurement with optimization of end-fire coupling," Elec. Lett. 28, 16121613 (1992).

6. G. D. Emmerson, S.P. Watts, C.B.E. Gawith, V. Albanis, M. Ibsen, R.B. Williams and P.G.R. Smith, "Fabrication of directly UV-written channel waveguides with simultaneously defined integral Bragg gratings,” Elec. Lett. 38, 1531-1532 (2002).

7. H.J.R. Dutton, "In-Fibre Bragg Gratings (FBGs)," in Understanding Optical Communications, (Prentice Hall PTR, 1998), pp. 266-267.

8. A.S. Webb, F.R.M. Adikan, J.K. Sahu, R.J. Standish, C.B.E. Gawith, J.C. Gates, P.G.R. Smith and D.N. Payne, "MCVD planar substrates for UV-written waveguide devices," Elec. Lett. 43, 517-519 (2007). 


\section{Figure Captions}

1. Schematic of sample produced for loss measurement. Two waveguides are direct UV written with ten and five integrated Bragg gratings, with central wavelengths as shown. The sample is $40 \mathrm{~mm}$ long with $2 \mathrm{~mm}$ long gratings, and $2 \mathrm{~mm}$ long waveguide sections between in the ten grating case, or $6 \mathrm{~mm}$ long separating sections in the waveguide with five gratings. Gratings are interrogated by launching from opposing directions.

2. (Color online) Obtained reflection spectra $\mathrm{R}^{\prime}$ and $\mathrm{R}^{\prime \prime}$ for waveguide containing ten Bragg gratings.

3. (Color online) Schematic showing the measured reflectivities $R_{i}{ }^{\prime}$ and $R_{i}{ }^{\prime \prime}$ from the $i^{\text {th }}$ grating in the waveguide. The input power reaching the grating (launched power minus coupling losses) from opposing directions is represented by $t$ and $t$.

4. Ratio of reflected power of each grating against the position of the grating within the waveguide. Plot is for waveguide containing ten gratings.

5. Ratio of reflected power of each grating against the position of the grating within the waveguide containing five gratings. 


\section{Figure 1}

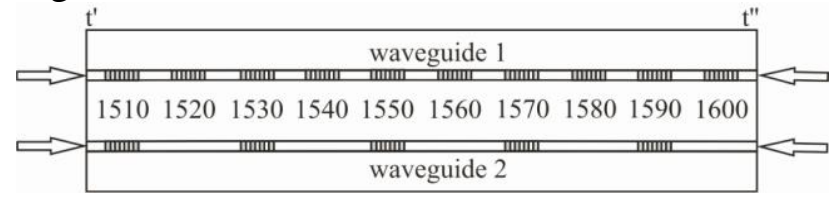

Figure 2

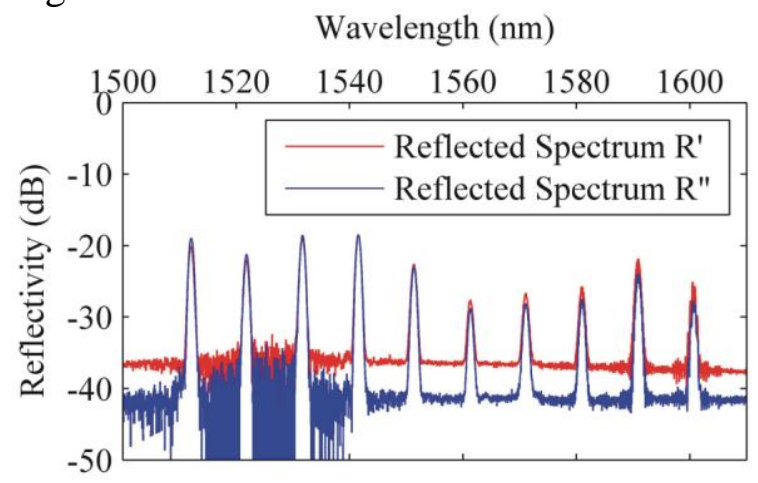

Figure 3

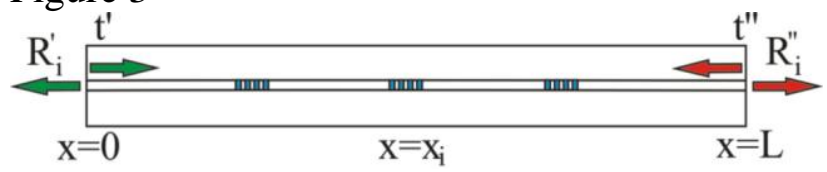

Figure 4

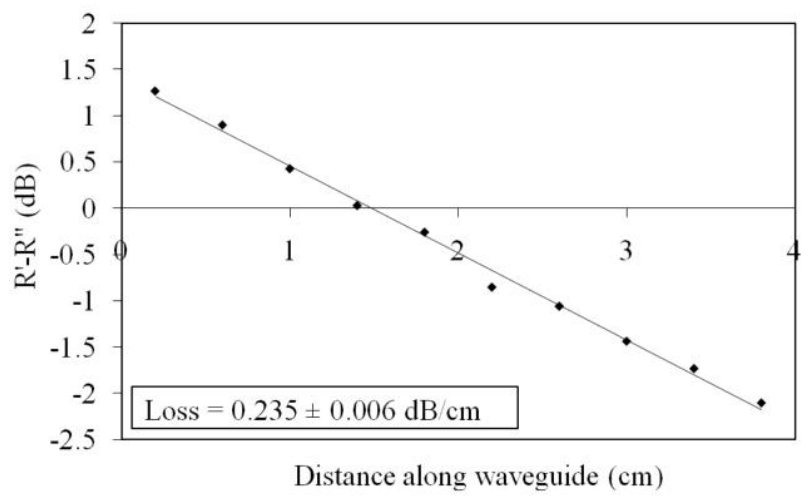


Figure 5

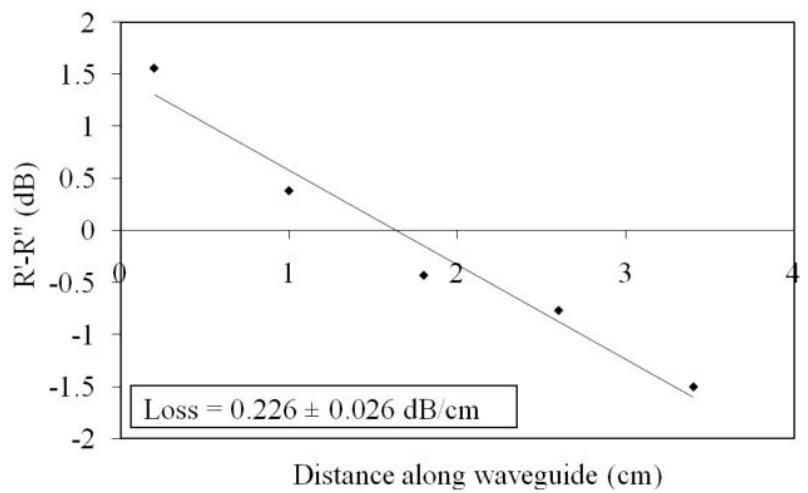

